\title{
FUNCIONES DE TRANSFORMACION PARA EVALUACIÓN DE IMPACTO POR ANTENAS DE ESTACIONES BASE TELEFONIA MOVIL
}

\section{TRANSFORMATION FUNCTIONS FOR EVALUATION OF IMPACT BY ANTENNAS OF BASE STATIONS MOBILE PHONE}

\author{
Msc(c). Alexander Armesto Arenas*, Msc(c). Wilson Angarita Castilla*, \\ $\mathrm{PhD}$. Sir Alexci Suarez Castrillón**
}
* Universidad Francisco de Paula Santander Ocaña, Investigador Grupo MINDALA. Vía Acolsure, Sede El Algodonal, Ocaña, Norte de Santander, Colombia. $(57+7) 5690088$. E-mail: \{aarmestoa, wangarita \}@ufpso.edu.co

**: Universidad Francisco de Paula Santander Ocaña, Investigador Grupo GRUCITE. Vía Acolsure, Sede El Algodonal, Ocaña, Norte de Santander, Colombia. $(57+7) 5690088$.

E-mail: sasuarezc@ufpso.edu.co

\begin{abstract}
Resumen: El presente estudio tuvo como objetivo definir métodos matemáticos mediante funciones de transformación que permitan medir la magnitud de las alteraciones ambientales provocadas por la instalación de estaciones base de telefonía móvil. Su aplicación fue realizada en el Colegio Instituto Técnico Industrial Ocaña. Para esto implementaron entrevistas, software QGIS y un equipo de medición de radiación electromagnética de alta frecuencia 60105 marca Aronia. Como resultado se obtuvieron cinco funciones de transformaciones en donde se relaciona el valor de impacto con las cantidades afectadas. En conclusión este tipo funciones son una herramienta que permite valora el impacto ambiental de este tipo de tecnologías de acuerdo a su magnitud. De acuerdo en su aplicación en el respectivo colegio, se concluye que hay un impacto ambiental adverso alto.
\end{abstract}

Palabras clave: Ambiente, antenas, funciones, impacto, telefonía

\begin{abstract}
This study have objective define mathematical methods using transformation functions to measure the magnitude of environmental changes caused by the installation of mobile phone base stations. In the implemention was used Interviews, software QGis and a High frequency spectrum analyzer HF 60105 Aronia. As a result were obtained five functions of transformations in which the impact value is related to the affected quantities In conclusion, these types of functions are a tool that allows assessing the environmental impact of these types of technologies according to their magnitude. According to their application in the respective college, it is concluded that there is a high adverse environmental impact.
\end{abstract}

Keywords: Antennas, Environment functions, impact, telephony.

\section{INTRODUCCIÓN}

En los indicadores de impacto ambiental se definen indicadores cualitativos y cuantitativos, los 
indicadores de carácter cuantitativo se pueden expresar numéricamente, este es el caso de los índices, en los cuales se requiere del uso de funciones de transformación y de técnicas de muestreo que permitan cuantificar o correlacionar las variables analizadas con el estado del factor ambiental (Prada, 2010). Este tipo de metodología permite medir la dimensión del impacto, teniendo muy en cuenta las cantidades de las partes afectadas.

En la función de transformación en el eje de las abscisas se ubica el índice o indicador y el eje de las ordenadas la magnitud de medida teniendo en cuanta que puede ser lineales o no, como pendientes negativas $\mathrm{o}$ positivas. (Alfonso Garmendia, 2008). Para el análisis de los impactos ambientales por la instalación de estaciones base de antenas de telefonía móvil, estas técnicas permiten valorar las alteraciones de la radiación de las antenas y de la misma forma otros factores relacionados con ella. De acuerdo a lo anterior, tengamos en cuenta que la radiación electromagnética es una combinación de emisión de ondas magnéticas y eléctricas que viajan a la velocidad de la luz (Barandiarán, 2003). Del mismo modo para la instalación de estas tecnologías, se debe tener en cuenta el uso del suelo (Comisión de Regulación de Comunicaciones. República de Colombia, 2012). Hay que mencionar a demás, que para su respectivo funcionamiento se deber tener en cuenta la participación de la comunidad que se puede ver afectada (Corte Constitucional de Colombia, 2015). Debido a que los niños y otras personas vulnerables pueden estar sobreexpuestos a los riesgos derivados de la contaminación por ondas electromagnéticas (Bergareche, 2013). Por otro lado hay que tener en cuenta que la fauna se puede ver afectada por la radiación electromagnética debido a que los seres vivos utilizan el campo magnético terrestres para orientarse (Tutivén) de esto se observa que los insectos se podrían ver afectados por este tipo de contaminación (Balmori, 2006), y de igual forma las ratas (Atasoy, Gunal, Atasoy, Elgun, \& Bugdayci., 2013). Con respecto a la flora, en un estudio realizado sobre las plantas de ají se observó que el campo electromagnético influye sobre el desarrollo de la planta (Carrejo, 2013), parecido el efecto lo tuvo el tomate (Angel de Souza, 199) y el arroz (Torres, 2008)

Todo lo anterior confirma, que para la puesta en marcha de la instalación y funcionamiento de antenas de estaciones base de telefonía móvil, no solamente hay que tener en cuenta la radiación electromagnética como fuente principal de las posibles alteraciones ambientales sobre los recursos naturales (flora y fauna), del mismo modo hay tener presente la participación de la ciudadanía y el aspecto socioeconómico y cultural que se pueda ver afectado. En consecuencia de esto y con el propósito de mejorar las metodologías de las evaluaciones de los posibles impactos ambientales adversos, este artículo tiene como objetivo definir métodos matemáticos mediante funciones de transformación que permitan medir la magnitud de las alteraciones ambientales y del mismo modo se pueda definir soluciones más relacionadas con cantidades las partes afectadas (Duran \& Iturriago., 2012, Moreno et al., 2013).

\section{METODOLOGIA}

El ensayo fue realizado desde el Laboratorio de Calidad del Aire de Universidad Francisco de Paula Santander Ocaña, y su implementación se ejecutó en la estación base de antenas de telefonía móvil instalada en el Colegio Instituto Técnico Industrial.

Para las mediciones se realizaron encuestas y visitas de campo. De acuerdo a las mediciones, se utiliza un analizador de espectro de alta frecuencia HF60105 Marca Aronia, con su respectivo certificado de calibración.

Para las funciones de transformación se tuvieron en cuenta ecuaciones trigonométricas y lineales.

El valor del impacto fue tomado como la variable dependiente (Tabla 1) en cada una de la funciones de transformación

Tabla 1. Variables

\begin{tabular}{|c|c|c|}
\hline \multirow{2}{*}{$\begin{array}{l}\text { Variable } \\
\text { dependiente }\end{array}$} & \multicolumn{2}{|c|}{ Variable independiente } \\
\hline & Variable & Unidad de medida \\
\hline \multirow{8}{*}{$\begin{array}{l}\text { Impacto } \\
\text { Ambiental (Ei) }\end{array}$} & $\begin{array}{l}\text { Radiación } \\
\text { electromagnética }\end{array}$ & $\begin{array}{l}\text { Watts/metro } \\
\text { cuadrado }\left(\mathrm{W} / \mathrm{m}^{2}\right)\end{array}$ \\
\hline & Distancia & Metros (m) \\
\hline & Área afectada & $\begin{array}{l}\text { Metros cuadrados } \\
\left(\mathrm{m}^{2}\right)\end{array}$ \\
\hline & Área Física & $\begin{array}{l}\text { Metros cuadros }\left(\mathrm{m}^{2}\right) \\
\text { del áreas física (Af) }\end{array}$ \\
\hline & Personas Afectadas & $\begin{array}{ll}\text { Número } & \text { de } \\
\text { personas }(\mathrm{Hi})\end{array}$ \\
\hline & Personas contratadas & $\begin{array}{ll}\text { Número } & \text { de } \\
\text { personas } & \\
\text { contratadas }(\mathrm{Pi}) & \end{array}$ \\
\hline & $\begin{array}{l}\text { Especies de plantas } \\
\text { afectadas }\end{array}$ & $\begin{array}{l}\text { Número de especies } \\
\text { afectadas }\left(S_{i, v)}\right.\end{array}$ \\
\hline & $\begin{array}{l}\text { Especies de animales } \\
\text { afectados }\end{array}$ & $\begin{array}{l}\text { Número de especies } \\
\text { de animales } \\
\text { afectados }\left(\mathrm{S}_{\mathrm{i}, \mathrm{a}}\right)\end{array}$ \\
\hline
\end{tabular}


Todas las funciones fueron representadas en planos cartesianos, describiendo en el eje de las ordenadas el valor del impacto y en el eje de las abscisas la variable independiente.

De acuerdo a la calificación del impacto este fue hallado con relación a lo explicado en la tabla 2 .

Tabla 2: Valoración de impactos adversos

\begin{tabular}{|c|c|}
\hline Valor & Calificación \\
\hline$(0-0.2)$ & Muy bajo \\
\hline$[0.2-0.4)$ & Bajo \\
\hline$[0.4-0.6)$ & Medio \\
\hline$[0.6-0.8)$ & Alto \\
\hline$[0.8-1)$ & Muy alto \\
\hline
\end{tabular}

\section{RESULTADOS}

Se hallaron hallaron cinco (5) funciones de transformación donde se relacionan en el Impacto con cada una de las variables independientes anteriormente nombradas.

\subsection{Impacto - Radiación electromaganeticas}

Los niveles de radiación electromagnética de telefonía móvil son muy pequeños, y el aumento del impacto en relación con su intensidad es muy lento.

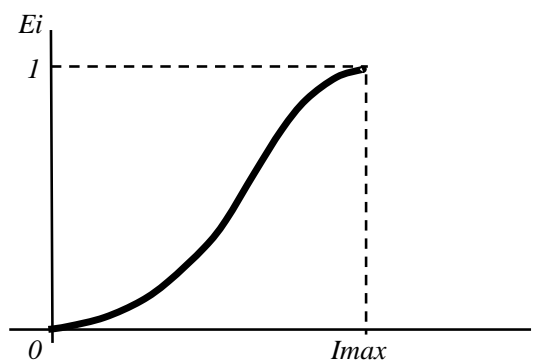

Fig. 1. Evaluación del impacto en relación con la intensidad de radiación

La función de transformación acorde con la anterior grafica se demuestra como:

$E i=\frac{-\cos \left(\frac{\pi I}{\operatorname{lmax}}\right)+1}{2} 0 \leq I \leq \operatorname{Imax}$

Ei: Impacto ambiental

I: intensidad de radiación electromagnética medida $\left(\mathrm{W} / \mathrm{m}^{2}\right)$

Imax: Intensidad máxima permitida

\subsection{Impacto - Distancia de ubicación de la antena}

Con respecto a la distancia de la antena y el receptor, en los países han definido trayectos mínimos entre las dos partes. De acuerdo a esto se define la siguiente gráfica, teniendo en cuenta que la diminución del impacto nunca llegará a tener el valor de cero.

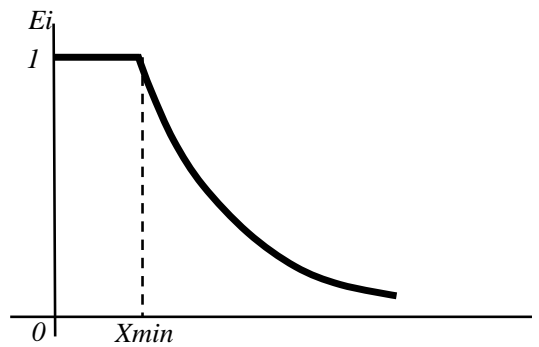

Fig. 1. Evaluación del impacto en relación con distancia de ubicación de la antena

La función de transformación:

$E i=1 \quad 0 \leq x \leq X \min$

$E i=\frac{x_{\min }}{x_{\min }+x} \quad x>X \min$

$x_{\text {min }}$ : Distancia mínima permitida en metros $(m)$ $x$ : distancia medida a partir de la distancia mínima

\subsection{Impacto - Área afectada}

Con relación al área de la flora, fauna y área física $\left(\mathrm{A}_{\mathrm{f}}\right)$ afectada, se tendrá la relación directa entre el área afectada y el área total existente.

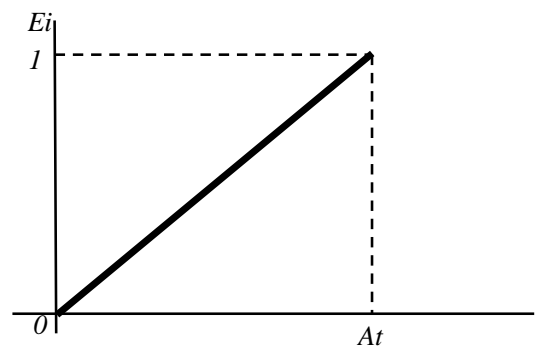

Fig. 1. Evaluación del impacto en relación con el área afectada

Función de transformación:

$E i=\frac{1}{A_{\mathrm{t}}} * A_{\mathrm{i}} \quad A_{\mathrm{i}} \leq A_{\mathrm{t}}$ 
$\mathrm{A}_{\mathrm{i}}=$ Área impactada en metros cuadrados $\left(\mathrm{m}^{2}\right)$ $\mathrm{A}_{\mathrm{t}}=$ Área total

\subsection{Impacto - número de personas y especies afectadas}

Teniendo presente el número de personas y de especies de flora y fauna afectada directamente por la antena, se tendrá la relación de los individuos que habitan dentro de la distancia mínima de distancia permitida.

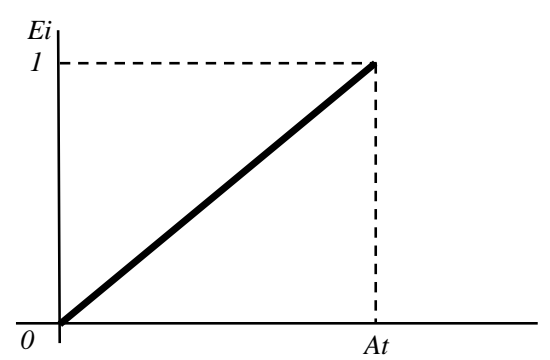

Fig.1. Evaluación del impacto en relación con el número de personas y de especies de flora y fauna impactadas

Función de transformación:

$E i=\frac{1}{H_{t}} * H_{\mathrm{i}} \quad H_{\mathrm{i}} \leq H_{\mathrm{t}}(4)$

$H_{t}$ : Número de Habitantes o de especies totales de área de estudio

$H_{i}$ : Número de Habitantes o de especies impactados

Nota: Para el número de especies de plantas se determinará con el símbolo $S_{\mathrm{i}, \mathrm{v}}$, y para el número de especies de animales $S_{\mathrm{i}, \mathrm{a}}$

\subsection{Impacto - beneficio económico}

Acerca del beneficio económico que brinda el funcionamiento de las antenas se define el número de personas contratas que habitan la zona impactada.

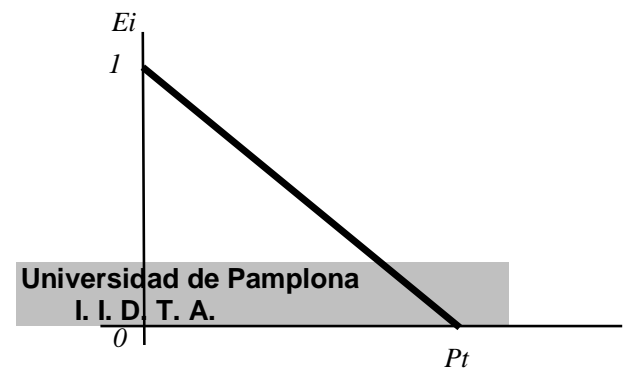

Fig. 1. Evaluación del impacto en relación con el número de personas contratadas

$$
E i=-\frac{1}{P_{t}} * P_{\mathrm{i}}+1 \quad P_{\mathrm{i}} \leq P_{\mathrm{t}}
$$

$P_{i}$ : Número de Personas contratadas

$P_{t}$ : Número de personas totales con posibilidad de trabajo.

Para definir el grado del impacto de determino el mismo nivel de importancia para cada una de las partes afectadas.

El cálculo total del impacto se determina como la sumatoria de todos los impactos hallados

$$
\text { Ei total }=\sum_{i=1}^{n} x_{i}
$$

\section{$x_{i}$ : Valor del impacto}

Para su valoración se determinó la relación entre la sumatoria total de los impactos adversos y el valor máximo que puede alcanzar dicha sumatoria

$$
\text { Valoración }=\frac{\sum_{i=1}^{n} x_{i}}{8}(7)
$$

\subsection{Implementación del modelo de las funciones de transformación}

Para el ejemplo de aplicación de las anteriores funciones, se tomó el colegio Instituto Técnico Industrial de la Ciudad Ocaña. Colombia. Presentando como resultado un valor de 0.62, calificado como un impacto ambiental adverso alto

$\underline{\text { Tabla 2: Evaluación de impacto ambiental }}$

\begin{tabular}{ccccc}
\hline Variable & $\begin{array}{c}\text { Intensidad } \\
\text { permitida }\end{array}$ & $\begin{array}{c}\text { Intensidad } \\
\text { medida }\end{array}$ & Ei & Calificación \\
\hline I & 9.5 & $2.45 \times 10^{-7}$ & 0.0 & Muy bajo \\
& $\mathbf{Q}$ & $\mathbf{Q}_{\mathbf{i}}$ & & \\
\hline
\end{tabular}




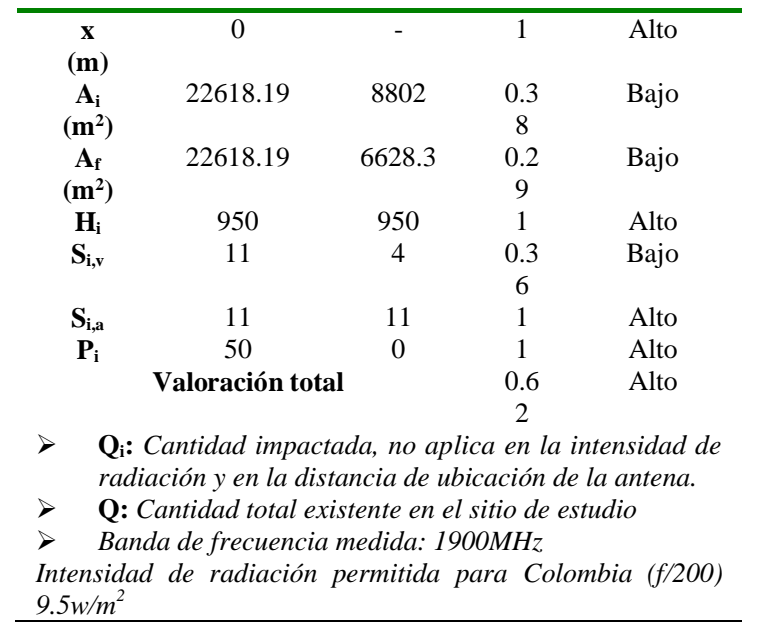

\section{CONCLUSIONES}

Las funciones de transformación permiten definir la valoración del impacto de acuerdo a las cantidades impactadas, lográndose un análisis menos subjetivo y más ajustado a la realidad y magnitud del entorno afectado.

De acuerdo a la aplicación realizada en el Colegio Instituto Técnico Industrial Ocaña. Se concluye que a pesar que el impacto adverso por la emisión de radiación fue muy bajo, no se tuvo en cuenta los demás componentes, dando como resultado final un impacto ambiental adverso alto.

\section{RECONOCIMIENTO}

Agradecimiento a la Universidad Francisco de Paula Santander Ocaña, y en especial a la Facultad de Ciencias Agrarias y del Ambiente y al Departamento de Investigación y Extensión por el apoyo a la exploración de nuevos avances tecnológicos

\section{REFERENCIAS}

Garmendia, A. S. (2008). Enseñanza de la evaluación de impactos ambientales en escuelas técnicas. Una reflexión sobre las funciones de transformación. Enseñanza de la evaluación de impactos ambientales en escuelas técnicas. Una reflexión sobre las funciones de transformación (págs. 380-382). Valencia: Instituto de Ciencias de la Educación. Universidad Politécnica de Valencia.

Ángel de Souza T, R. C. (199). Efecto del tratamiento magnético de semillas de tomate (lycopersicon esculentum mill) sorre la germinacion y crecimiento de las plantulas. invest. agr., 439-444.

Atasoy, H. I., Gunal, M. Y., Atasoy, P., Elgun, S., \& Bugdayci., G. (2013). Demostración inmunohistofisiológica de efectos deletéreos en los test de ratas en crecimiento de ondas de radiofrecuencia emitidas por dispositivos Wi-Fi convencionales. Journal Pediatric Urology, 223-229.

Balmori, A. (2006). Efectos de las Radiaciones Electromagnéticas de Telefonia Movil sobre los Insectos. Ecosistemas, 87-95.

Bergareche, R. L. (2013). Contaminación por radiación electromagnética en personas vulnerables: tutela preventiva y generación de otras fuentes de energía. Actualidad Jurídica.

Carrejo, M. M. (2013). Conclusiones. Tesis, universidad del valle, escuela de ingeniería de los recursos naturales y del ambiente - Eidenar.

Durán Acevedo Christian M, Iturriago Ali Xavier. (2012). Automatización de un Sistema de Suministro de Agua Potable a Través de la Tecnología Zigbee. Revista colombiana de tecnologías de Avanzada 1 (19), Pág. 36 - 42.

Moreno Rubio J., Jiménez López A, Barrera Lombana N. (2013). El amplificador de potencia de carga sintonizada. Revista colombiana de tecnologías de Avanzada. 2(22). Pág. 9 - 13.

Torres, J. E. (2008). Efecto de campos magnéticos en la germinación de semillas de arroz (Oryza sativa L.) y tomate (Solanum lycopersicum L.). Agronomía Colombiana, vol. 26, núm. 2, 2008, pp. 177-185, 26(2), 177-185.

Comisión de Regulación de Comunicaciones. República de Colombia. (2012). Clasificación del terreno. En C. d. Colombia, Código de Buenas Prácticas para el (pág. 53). Bogotá.

Corte Constitucional de Colombia. (2015). Artículo 79. En Constitución Política (pág. 72). Bogotá.

Barandiarán García, J.M. (2003). Maxwell y la propagación. En J. M. García, El Magnetismo en la Vida Cotidiana (págs. 1-22). Bilbao: Real Sociedad Bascongada de los Amigos del País.

Prada, R. J. (2010). Indicadores de Impacto Ambiental. Propuesta metodológica para la evaluación de impacto. Bogotá, Colombia. 